\title{
Symmetry Reductions in Model Checking *
}

\author{
E. M. Clarke ${ }^{1}$ and E. A. Emerson ${ }^{2}$ and S. Jha ${ }^{1}$ and A.P. Sistla ${ }^{3}$ \\ 1 School of Computer Science, Carnegie Mellon University, Pittsburgh, PA \\ 2 Department of Computer Science, University of Texas, Austin, TX \\ 3 Department of Electrical Engg and Computer Science, University of Illinois, \\ Chicago, IL
}

\begin{abstract}
The use of symmetry to alleviate state-explosion problems during model-checking has become a important research topic. This paper investigates several problems which are important to techniques exploiting symmetry. The most important of these problems is the orbit problem. We prove that the orbit problem is equivalent to an important problem in computational group theory which is at least as hard as the graph isomorphism but not known to be $N P$-complete. This paper also shows classes of commonly occurring groups for which the orbit problem is easy. Some methods of deriving symmetry for a shared variable model of concurrent programs are also investigated. Experimental results providing evidence of reduction in state space by using symmetry are also provided.
\end{abstract}

\section{Introduction}

Temporal Logic Model Checking is a technique for determining whether a temporal logic formula is valid in a finite state system $M=(S, R, L)$, where $S$ is the state space, $R$ is the state transition relation, and $L$ is a function that labels states with sets of atomic propositions [4]. Such a structure is usually called a Kripke structure and may have an enormous number of states because of the state explosion problem. A system with $n$ boolean state variables can have a state space which is exponential in $n$. An efficient Model Checking procedure tries to reduce the number of states that are actually explored $[2,4]$. Recently, techniques which exploit the inherent symmetry of the system while performing model checking have become quite popular $[3,6,11]$.

Basically, the idea of exploiting symmetry is the following: given a Kripke Structure $M=(S, R, L)$, a symmetry group $G$ is a group acting on the state set $S$ that preserves the transition relation $R, G$ partitions the state set $S$ into equivalence classes called orbits. A quotient model $M_{G}$ is constructed that contains one or more representative from each orbit. The state space $S_{G}$ of the

\footnotetext{
* Clarke and Jha's research was supported in part by NSF Grant no. CCR-8722633 and SRC Contract 92-DJ-294; Emerson's research was supported in part by NSF grant no. CCR-941-5496 and SRC grant no. 388-DP-97; Sistla's research was supported in part by NSF grants CCR-9623229 and CCR-9633536.
} 
quotient model will, in general, be much smaller than the original state space $S$. This makes it possible to verify much larger structures.

This paper investigates several problems associated with exploiting symmetry. For example, given a group $G$ and two states $s$ and $s^{\prime}$, the orbit problem asks whether $s$ and $s^{\prime}$ are in the same orbit. Determining whether two states are in the same orbit is at the core of any model checking procedure exploiting symmetry. We prove that the orbit problem is equivalent to an important problem in computational group theory. We explore ways of deriving symmetries of shared variable concurrent programs. Since the orbit problem in its full generality is quite hard, this paper also shows that the orbit problem is easy for certain commonly occurring groups and provides alternative techniques which do not require solving the orbit problem. We have also built a model-checker called SYMM which allows the user to specify the symmetries of the model and then uses the symmetries to perform efficient model-checking. Few of these problems were considered in $[3,6]$. The results provided in this paper represent significant advances over those presented in $[3,6]$. Although the paper only presents results for asynchronous composition of processes, all the results do extend to synchronous composition of processes but are not presented here because of lack of space. Complete details will be provided in the full version of the paper.

Our paper is organized as follows: In Section 2 we introduce a shared variable model. Section 3 gives a technique to derive symmetries of shared variable programs. Section 4 investigates the complexity of the orbit problem. Section 5 investigates some special classes of the orbit problem. Experimental results on an arbiter example are shown in section 6 . Section 7 concludes with some interesting future directions. Due to space limitations we have not presented the background material here. For background on group theory the reader is referred to [9]. For general background on symmetry groups and model-checking see $[3,6]$.

\section{A Shared Variable Model of Computation}

We adopt the model of computation from [6]. A shared variable program is defined with the state sets and the transition relation as follows:

- $S=L o c^{I} \times D^{V}$ is the finite set of states, with $L o c$ a finite set of individual process locations, $I$ the set of process indices, and $V$ is a finite set of shared variables over a finite data domain $D$.

$-R \subseteq S \times S$ which represents the transitions of the system.

For convenience, each state $s=\left(s^{\prime}, s^{\prime \prime}\right) \in S$ can be written in the form $\left(\ell_{1}, \ldots, \ell_{n}, v=d, \ldots, v^{\prime}=d^{\prime}\right)$ indicating that processes $1, \ldots, n$ are in locations $\ell_{1}, \ldots, \ell_{n}$, respectively and the shared variables $v, \ldots, v^{\prime}$ are assigned data values $d, \ldots, d^{\prime}$, respectively.

Next, we define a labeling function for a shared variable program. The set of terms are expressions of the form $l_{i}(i \in I)$ and $v=d(v \in V$ and $d \in D)$. The set of atomic propositions $A P$ are constructed from the set of terms by the 
logical connectives $\wedge$ and $\neg$. Given an atomic proposition $p \in A P$ and a state $s \in S$, the satisfaction relation $s \models p$ is defined in the following way:

$s \models l_{i}$ iff the $i$-th process in the state $s$ is in location $l_{i}$.

$s \models(v=d)$ iff the shared variable $v$ has the value $d$ in the state $s$.

$s \vDash f \wedge g$ iff $s \models f$ and $s \models g$.

$s \vDash \neg f$ iff $s \not \models f$.

Given a shared variable program, we can construct a corresponding Kripke Structure $M=(S, R, L)$ ( $S$ and $R$ were defined before) by constructing the following labeling function $L: S \rightarrow 2^{A P}: p \in L(s) \Leftrightarrow s \models p$.

In practice, for ordinary model checking, $M$ is the Kripke Structure corresponding to a finite state concurrent program $\mathcal{P}$ of the form $\|_{i=1}^{n} K_{i}$ consisting of processes $K_{1}, \ldots, K_{n}$ running in parallel. Each $K_{i}$ may be viewed as a finite state transition graph with node set Loc. An arc from node $\ell$ to node $\ell^{\prime}$ may be labeled by a guarded command $B \rightarrow A$. The guard $B$ is an atomic proposition that can inspect shared variables and local states of "accessible" processes. $A$ is a set of simultaneous assignments to shared variables $v:=d\|\cdots\| v^{t}:=d^{\prime}$. When process $K_{i}$ is in local state $\ell$ and the guard $B$ evaluates to true in the current global state, the program $\mathcal{P}$ can nondeterministically choose to advance by firing this transition of $K_{i}$ which changes the local state of $K_{i}$ to be $\ell^{\prime}$ and the shared variables in $V$ according to $A$. Thus the arc from $\ell$ to $\ell^{\prime}$ in $K_{i}$ represents a local transition of $K_{i}$ denoted by $\ell: B \rightarrow A: \ell^{\prime}$.

The Kripke structure $M=(S, R, L)$ corresponding to $\mathcal{P}$ is thus defined using the obvious formal operational semantics. First, the set of (all possible) states $S$ is determined from $\mathcal{P}$ because it provides us with the set of local (i.e., individual process) locations $L o c$, process indices $I$, variables $V$, and data domain $D$. For states $s, t \in S$, we define $s \rightarrow t \in R$ iff $\exists i \in I$ such that the process $K_{i}$ can cause $s$ to move to $t$, denoted by $s \rightarrow_{i} t$ iff $\exists i \in I \quad \exists$ local transition $\tau_{i}=\ell_{i}$ : $B_{i} \rightarrow A_{i}: m_{i}$ of $K_{i}$ which drives $s=\left(s^{\prime}, s^{\prime \prime}\right)$ to $t=\left(t^{\prime}, t^{\prime \prime}\right) ; i$-th component of $s^{\prime}$ equals $\ell_{i}$, the $i$-th component of $t^{\prime}$ equals $m_{i}$, all other components of $s^{\prime}$ equal the corresponding component of $t^{\prime}$, predicate $B_{i}(s)=$ true, and $t^{\prime \prime}=A_{i}\left(s^{\prime \prime}\right) . A_{i}\left(s^{\prime \prime}\right)$ is constructed from $s^{\prime \prime}$ by replacing the values of the shared variables according to the simultaneous assignment statement $A_{i}$. The labeling function $L$ is defined as before. Notice that we use asynchronous composition in the definition given here. Analogous definition can be given for synchronous composition. All the results given in the paper hold for synchronous composition, but are not stated because of lack of space.

\section{Deriving Symmetry}

This section analyzes how one can derive symmetry for shared variable programs introduced in the previous section. Intuitively, if one has a graph $G$ whose nodes corresponds to processes and the processes communicate over the edges of $G$, an automorphism of the graph $G$ should manifest itself into a symmetry of the underlying structure $[3,6]$. Succinctly speaking, structural symmetry introduces 
symmetry in the model [3](cf. [5]). This section proves that for certain cases one can derive the symmetry of the model from the topology of the system. Given a concurrent program $\mathcal{P}=\|_{i=1}^{n} K_{i}$, we build a hypergraph $H G(\mathcal{P})$. Under certain restrictions, we prove that each automorphism of $H G(\mathcal{P})$ is also a symmetry of the underlying Kripke Structure $M$. A restricted version of the theorem already appeared in [6]. There the authors assumed that all processes are isomorphic and the variables are only shared between two processes. The new and more general version of the theorem, presented here, can handle a broader class of systems. For example, an arbiter which maintains a global shared variable (indicating who has the resource) can now be accomodated.

Let $\mathcal{P}=\|_{i=1}^{n} K_{i}$ be a concurrent program. In this section the index set is $I=[n]$. Each shared variable $v$ is subscripted by the set of indices of the processes which access that shared variable. For example, if $x$ is accessed by processes 1,4 , and 5 , we write $x$ as $x_{\{1,4,5\}}$. Notice that each shared variable is uniquely determined by its name and subscript, but we allow shared variables to have the same name as long as their subscripts are different. For example, $x_{\{1,2\}}$ and $x_{\{3,4\}}$ are allowed. A permutation $\pi \in S_{n}$ acts on the variables in a natural manner, i.e., $\pi\left(x_{w}\right)=x_{\pi(w)}$. A permutation $\pi$ acting on $[n]$ is called consistent if and only if for every shared variable $x_{w}, x_{\pi(w)}$ is a variable as well. This means that we only allow permutations which map shared variables to shared variables.

We define how a consistent permutation $\pi$ acts on states, atomic propositions, and processes. Let $\pi$ be a consistent permutation.

- Given a state $s=\left(l_{1}, \cdots, l_{n}, v_{w_{3}}=d_{1}, \cdots, v_{w_{k}}=d_{k}\right)$, the state $\pi(s)$ is defined as follows: $i$-th process is in location $l_{\pi(i)}$ in the state $\pi(s)$, and the shared variable $v_{\pi(w)}$ in the state $\pi(s)$ has the same value as the variable $v_{w}$ in the state $s$.

- Let $p \in A P$ be an atomic proposition. $\pi(p)$ is recursively defined as follows: $\pi(f \wedge g)=\pi(f) \wedge \pi(g), \pi(\neg f)=\neg \pi(f), \pi\left(l_{i}\right)=l_{\pi(i)}$, and $\pi\left(v_{w}=d\right)=$ $\left(v_{\pi(w)}=d\right)$.

- Given a simultaneous assignment $A=\left(v_{w_{1}}=d_{1}\|\cdots\| v_{w_{k}}=d_{k}\right)$, define $\pi(A)$ as the following simultaneous assignment: $v_{\pi\left(w_{1}\right)}=d_{1}\|\cdots\| v_{\pi\left(w_{k}\right)}=d_{k}$

- Given a process $K_{i}$, the process $\pi\left(K_{i}\right)$ is constructed in the following manner: $l: B \rightarrow A: l^{\prime}$ is a transition in $K_{i}$ iff $l: \pi(B) \rightarrow \pi(A): l^{\prime}$ is a transition in $\pi\left(K_{i}\right)$.

Definition 1. A colored hypergraph with $n$ vertices and $k$ colors is a 3-tuple $H=([n], E, L)$ such that $E \subseteq 2^{[n]}$ is the edge set, and $L:[n] \rightarrow[k]$ is the coloring function which colors each node with one of the $k$ colors. A permutation $\pi$ acting on $[n]$ is called an automorphism of the hypergraph $H$ iff the following two conditions hold:

For all $1 \leq i \leq n, L(i)=L(\pi(i))$.

$w \in E$ iff $\pi(w) \in E$.

The group of automorphisms of the hypergraph $H$ is denoted by $\operatorname{Aut}(H)$.

Given a process $K_{i}$, let $\tau\left(K_{i}\right)$ be the type of that process. For example, if process $K_{i}$ is an instance of MODULE $m$ [14], then $\tau\left(K_{i}\right)=m$. Next, we define the 
concept of isomoprhism between two processes. Let $\Gamma\left(K_{i}\right)$ be the set of indices $r$ such that there exists a shared variable $x_{w}$ such that $\{i, r\} \subseteq w$. Intuitively, if $r \in$ $\Gamma\left(K_{i}\right)$, then $K_{i}$ and $K_{r}$ share some variables. $\Gamma\left(K_{i}\right)$ is called the neighborhood of $K_{i}$. We require that $i \in \Gamma\left(K_{i}\right)$, i.e., a process is in its own neighborhood. We say that $K_{i} \cong K_{j}$ if and only if for every consistent permutation $\pi$ such that $\pi(i)=j$ and such that for all $r \in \Gamma\left(K_{i}\right), \tau\left(K_{r}\right)=\tau\left(K_{\pi(r)}\right)$ it is the case that $\pi\left(K_{i}\right)=K_{j}$ and vice versa. Many times the condition $K_{i} \cong K_{j}$ can be checked by checking that $\pi\left(K_{i}\right)=K_{j}$ with respect to all permutations $\pi$ that map $i$ to $j$ and that map elements in $\Gamma\left(K_{i}\right)$ to elements in $\Gamma\left(K_{j}\right)$ and that satisfy local consistency conditions (i.e. $x_{w}$ is a variable iff $x_{\pi(w)}$ is a variable for cases where $i \in w)$; this property can be checked efficiently.

Definition 2. Given a concurrent program $\mathcal{P}=\|_{i=1}^{n} K_{i}$, define the corresponding colored hypergraph $H G(\mathcal{P})=([n], E, L)$ in the following manner:

$-w \in E$ iff there exists a shared variable with subscript $w$.

- Partition the processes $K_{1}, \cdots, K_{n}$ into equivalence classes induced by the relation $\cong$. Let $c_{1}, \cdots, c_{k}$ be the $k$ equivalence classes. The coloring function $L$ is defined as follows: $L(i)=r$ iff the process $K_{i}$ is in the equivalence class $c_{r}$.

Theorem 3. Let $H G(\mathcal{P})$ be the hypergraph corresponding to the program $\mathcal{P}=$ $\|_{i=1}^{n} K_{i}$. Let $M$ be the Kripke Structure corresponding to $\mathcal{P}$. Given these conditions, $\operatorname{Aut}(H G(\mathcal{P})) \leq \operatorname{Aut}(M)$.

\section{Complexity of the Orbit Problem}

In this section we assume that the state space of our system is given by assignments to $n$ boolean state variables $x_{1}, \cdots, x_{n}$. Therefore, the state space is isomorphic to $B^{n}$ (where $B=\{0,1\}$ ). We assume that the symmetry group $G \leq S_{n}$ acts on $B^{n}$ in the natural way: a permutation $\sigma$ maps a vector $\left(z_{1}, \cdots, z_{n}\right)$ to $\left(z_{\sigma(1)}, \cdots, z_{\sigma(n)}\right)$. The orbit problem is at the core of any method exploiting symmetry $[3,6,12,11]$. The orbit problem asks whether two states $s$ and $s^{\prime}$ (which in this case are two $0-1$ vectors of size $n$ ) are in the same orbit, i.e., there exists a permutation $\sigma \in G$ such that $s^{\prime}=\sigma(s)$. In [3] it was proved that the graph isomorphism problem can be reduced to the orbit problem. Therefore, the orbit problem is atleast as hard as the graph isomorphism problem. Here we show that the orbit problem is equivalent to the problem of finding a set stabilizer of a set $Y$ in a coset (we call this problem $S S C$ ). Since the graph isomorphism problem can be reduced to $S S C$ [8], this result subsumes the result which appeared in [3]. Moreover, $S S C$ (and hence the orbit problem) is equivalent to several important problems in computational group theory, which are harder than graph isomorphism, but not known to be $N P$-complete. Proofs of most the theorems are based on techniques introduced in [13].

The Orbit Problem (OP): Given two 0-1 vectors $x$ and $y$ of size $n$ and a 
group $G \leq S_{n}$, does there exist a permutation $\sigma \in G$ which maps $x$ to $y$, i.e., $y=\sigma(x)$.

Set Stabilizer in a coset $(S S C)$ : Given a set $Y \subseteq[n]$, let $G \leq S_{n}$ be a group and $\gamma \in S_{n}$ be a permutation. The problem is to find whether there exists $\sigma \in G \gamma$ which stabilizes the set $Y$, i.e., $\sigma(Y)=Y$.

Constructive Set Stabilizer in a coset $(C S S C)$ : Given a set $Y \subseteq[n]$, let $G \leq S_{n}$ be a group and $\gamma \in S_{n}$ be a permutation. The problem is to find whether there exists $\sigma \in G \gamma$ which stabilizes the set $Y$, i.e., $\sigma(Y)=Y$ and if yes, to exhibit such a $\sigma$.

Lemma 4. The problems $S S C$ and $C S S C$ are polynomially equivalent.

Theorem 5. The problems $O P$ and $S S C$ are polynomially equivalent.

In general, the $S S C$ problem is harder than graph isomorphism [8]. Conditions under which $S S C$ can be solved in polynomial time are discussed in $[8,13]$. In [8] it is proved that the Coset Intersection Emptiness problem stated below is polynomially equivalent to several important problems in computational group theory.

Definition 6. Coset Intersection Emptiness (CIE) Given the groups $A, B \leq$ $S_{n}$ by generating sets and given a permutation $\pi \in S_{n}$, test whether $A \pi \cap B$ is empty.

Theorem 7. The set stabilizer in a coset problem $(S S C)$ is polynomially equivalent to CIE.

Therefore, using the previous theorems one can deduce that $O P$ is polynomially equivalent to CIE and hence several problems in computational group theory.

\subsection{The Constructive Orbit Problem}

Modeling states by boolean variables, in some cases, is too cumbersome and detailed. For example, consider the shared variable program introduced in Section 2. Let $\mathcal{P}=\|_{i=1}^{n} K_{i}$ be a concurrent program which does not have shared variables. Let the size of the set of locations $L o c$ be $k$. In this case, a typical state in $\mathcal{P}$ is given by a vector of size $n$ whose elements are integers between 1 and $k$, i.e., the space $[k]^{n}$. Permuting the processes $K_{i}$ amounts to permuting the corresponding integers in that state. A symmetry group $G \leq S_{n}$ acts on the space $[k]^{n}$ in the following way: a permutation $\sigma \in G \operatorname{maps}\left(x_{1}, \cdots, x_{n}\right)$ to $\left(x_{\sigma(1)}, \cdots, x_{\sigma(n)}\right)$.

Given a symmetry group $G$, one frequently needs a representative function $\xi: S \rightarrow S[3,6,11]$ ( $S$ is the state space of the system) which has the following properties:

- $s$ and $\xi(s)$ are in the same orbit.

- If $s$ and $s^{\prime}$ are in the same orbit, then $\xi(s)=\xi\left(s^{\prime}\right)$. 
Such a representative function is used during state exploration in $[3,6,11]$. The need to find such a representative function motivates the following problem.

Definition 8. The Constructive Orbit Problem (COP): Given a group acting on $[n]$ and vector $x=\left(x_{1}, \cdots, x_{n}\right)$ find the lexicographically least element (or lex-least element for short) in the orbit of $x$ (the group $G$ permutes the indices of $x$ )

Notice that if one can solve $C O P$ in polynomial time, one can construct the representative function $\xi$. Given a state $x, \xi(x)$ is simply the lex-least element in its orbit. In [1] it is proved that the problem is $N P$-hard. The paper also shows that for certain special groups $C O P$ can be solved in polynomial time. Actually, for our purposes it is enough to find a canonical element from each orbit.

\section{Working Around the Orbit Problem}

Results of section 4 prove that the Orbit problem and the Constructive Orbit Problem are quite hard in its full generality. In this section we discuss two possible techniques which will help circumvent the complexity of the orbit problem.

1. We prove that for a large class of groups, which occur commonly in practice, the orbit problem can be easily solved. This means that if the symmetries are restricted to this class of groups, the orbit problem can be easily solved.

2. We also describe an approach that uses multiple representatives from each orbit rather than just one. This approach is partcularly useful for symbolic model checking [3].

The ensuing subsections outline these approaches.

\subsection{Easy Groups}

Notice that if a group $G \leq S_{n}$ has polynomial size, $C O P$ for $G$ can be solved in polynomial time by exhaustive enumeration. For example, a rotation group acting on set of size $[n]$ has order $n$. Therefore, for the rotation group one can solve $C O P$ in linear time. The lemma given below states that if $C O P$ can be solved in polynomial time for two disjoint groups $J$ and $K$, then $C O P$ can be solved in polynomial time for their direct product.

Lemma 9. Let $G$ be a disjoint product of $J$ and $K$. If $C O P$ for $J$ and $K$ can be solved in polynomial time, then $C O P$ for $G$ can be solved in polynomial time.

The next lemma is similar to the previous one but refers to wreath products.

Lemma 10. Let $G=J \imath K$ be the wreath product of $J$ and $K$. The group $J, K, G$ act on the sets $[n],[m],[n m]$ respectively. If $C O P$ for $J, K$ can be solved in polynomial time, then $C O P$ for $G$ can be solved in polynomial time. 
Lemma 11. Let $S_{n}$ be the full symmetric group acting on the set [n]. The COP problem for $S_{n}$ can be solved in polynomial time.

Proof: Given a vector $x=\left(x_{1}, \cdots, x_{n}\right)$, the lex-least element of $x$ under the group $S_{n}$ can be obtained by sorting the elements $x_{i}$.

In practice, symmetries are given as a set of transpositions. For example, in a system which has the star topology, the two outer processes can be switched. The lemma given below states that if the group is only generated by transpositions, then $C O P$ for it can be solved in polynomial time.

Lemma 12. Let $G$ be a permutation group acting on the set $[n]$. Assume that $G$ is generated by a set of transpositions $S$. The $C O P$ problem for $G$ can be solved in polynomial time.

This means that during model-checking if one restricts to the class of groups mentioned above, exploiting symmetry is relatively easy. Hence while exploiting symmetry one should try to work with these easy groups.

\subsection{Multiple Representatives}

Multiple Representatives were discussed in [3]. The account given here is much cleaner and general. Assume that we are given a Kripke Structure $M=(S, R, L)$ and a symmetry group $G$ of $M$. Let $C \subseteq G$ be an arbitrary set of permutations which is inverse closed, i.e., $\pi \in C$ implies that $\pi^{-1} \in C$. We also assume that the identity permutation $e$ is in $C$. Let $R e p \subseteq S$ be a set of representatives which satisfies the following requirements:

1. Every orbit of $S$ under the action of $G$ has a non-empty intersection with Rep.

2. Given an $s \in S$, there exists a $\sigma \in C$ such that $\sigma(s) \in R e p$.

Let $M_{R e p}=\left(R e p, R_{R e p}, L_{R e p}\right)$, where $R_{R e p}$ and $L_{R e p}$ are defined as follows:

- We have that $\left(r_{1}, r_{2}\right) \in R_{R e p}$ iff there exists $s \in S$ and $\sigma \in C$ such that $\left(s, r_{2}\right) \in R$ and $\sigma(s)=r_{1}$.

- $L_{R e p}(r)=L(r)$.

The representative relation $\xi \subseteq S \times R e p$ is defined as follows: $(s, r) \in \xi$ iff there exists a $\sigma \in C$ such that $\sigma(s)=r$. Notice that $R_{R e p}=\eta^{-1} \circ R$.

Theorem 13. let $M=(S, R, L)$ be a Kripke structure, $G$ be a symmetry group of $M$, and $h$ be a $C T L^{\star}$ formula. If $G$ is an invariance group for all the atomic propositions $p$ occurring in $h$, then for all $r$ such that $(s, r) \in \xi$

$$
M, s \vDash h \Leftrightarrow M_{R e p}, r \models h
$$


A natural question to ask is: how does one choose Rep given a set of polynomial size $C$ ? One natural choice of $R e p$ is given below:

$$
r \in \operatorname{Rep} \Leftrightarrow \forall(\sigma \in C)(r \geq \sigma(r))
$$

where $\leq$ represents the lexicographical ordering on the $0-1$ vectors. It is easy to check that Rep has the required properties. Also notice that given an $s \in S$ and $r \in R e p$ one can decide the following questions in polynomial time?

1. Is $s$ a representative, i.e., $s \in R e p$ ?

2. Is $(s, r) \in \xi$ ?

First we argue that the choice of $C$ is very important on how much reduction is achieved. For example, let us consider the case when $C=G^{[1, k]}$. In this case we will only permute the first indices $k$ indices of the states. Therefore if there exists an orbit $\Theta \subseteq S$ which does not involve these $k$ indices, then $\Theta \subseteq R e p$, i.e., the whole orbit has to be represented. We need a way of choosing $C$ such that we have uniform savings across all orbits of $S$ in $G$. One possibility is to take a subgroup $H \leq G$ such that $[G: H]$ is polynomial in $n$. Let $B$ be the full right traversal of $H$ in $G$. Let $C$ be the inverse closure of $B$. The subgroup $H$ can be chosen in many ways and its choice will depend upon the structure of the system being verified. One choice is as follows: Fix a set $I=\left\{i_{1}, \cdots, i_{k}\right\} \subseteq[n]$ of size $k$. Let $H$ be the pointwize stabilizer of $I$ in $G$. The pointwise stabilizer of $I$ in $G$ is the following group:

$$
\{\sigma \in G \mid \forall(i \in I)(\sigma(i)=i)\}
$$

Notice that $[G: H] \leq n^{k}$. The subgroup $H$ and its full traversal in $G$ can be found in polynomial time [7].

\section{Empirical Results}

Using the multiple representatives theory presented in section 5.2 we have built a symbolic model checker called SYMM. The tool SYMM has a language based on the shared variable model of computation described earlier. It allows the user to give $C T L$ specifications. The tool also provides the facility for the user to give symmetries of the system. We have verified several examples using our tool. Here we describe our results for an arbiter example. In this arbiter, each module has a priority number. While competing for the bus, modules with higher priority number are given preference. Among competing modules with the same priority number, the winner is decided non-deterministically. Winner is the module which wins the competing phase. The arbitration cycle (which results in a winner) has six phases. We give a brief overview of these phases. The interested reader is referred to the IEEE Futurebus standard for a more detailed account [10].

Phase 0: In this phase modules decide to compete for the bus.

Phase 1: Noticing that a competition phase is about to begin, other modules might decide to compete. 
Phase 2: In this phase a winner (called the master elect) is selected from the set of competing modules. Winner is selected according to the rules described earlier.

Phase 3: Other modules check that the master elect had the highest priority number among the competing modules. If this is not the case, modules assert an error. If an error has not occurred, this phase continues until the master of the bus relinquishes its control. In this phase a module with higher priority than the master elect might start a new arbitration cycle. This is called deposing the master elect.

Phase 4: In this phase the current master of the bus might inhibit transfer of control of the bus to the master elect. If the master relinquishes its control over the bus, the arbitration cycle moves to the last phase.

Phase 5: In this phase the master elect gets control of the bus. This phase is called the transfer of tenure phase.

There are three boolean variables in each module which ensures the proper sequencing of the phases in an arbitration cycle. The internal state of the module depends on the outcome of the arbitration cycle and is shown below.

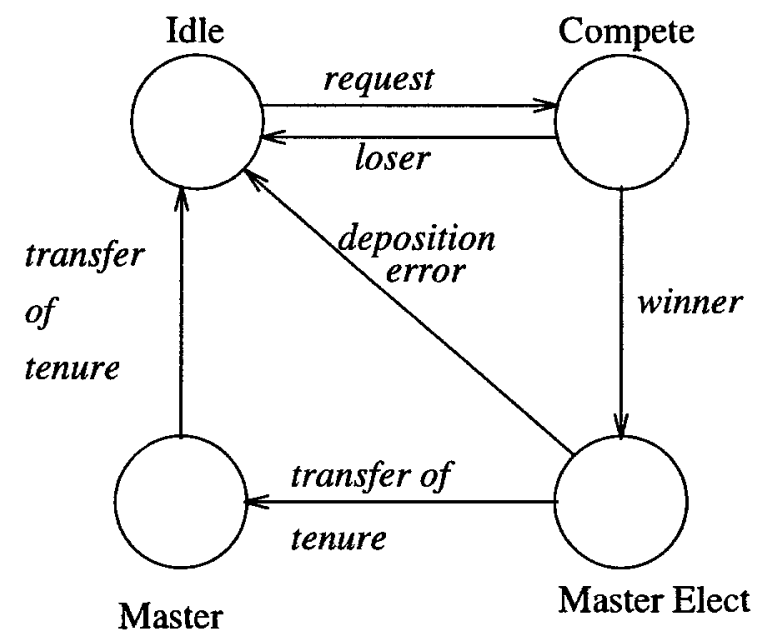

Fig. 1. States of the Arbiter

Notice that two modules with the same priority number can be permuted without changing the behavior of the system. Formally, the permutation corresponding to exchanging two modules with the same priority number is a symmetry of the system. However, two modules with different priority numbers cannot be exchanged. In the table given below the first column shows the configuration of the system. Assume that we have $m$ sets of $n$ modules (denoted by $\left.\left\{M_{1,1}, \cdots, M_{1, n}\right\}, \cdots,\left\{M_{m, 1}, \cdots, M_{m, n}\right\}\right)$. Moreover, the priority numbers of two modules $M_{j, l}$ and $M_{r, t}$ are the same iff $j=r$, or they belong to the same 
set. In this case the set of representatives $R e p$ is the set of states where the first module from a particular set is always chosen master elect. The set $C$ contains all the transpositions of the form $\sigma_{i, l}=((i, 1),(i, l))(1 \leq i \leq m$ and $1 \leq l \leq n)$ and their products. The transposition $\sigma_{i, l}$ corresponds to exchanging modules $M_{i, 1}$ and $M_{i, l}$. It is easy to see that $C$ is inverse closed. Moreover, given an arbitrary state $s$ there exists a representative state $r \in R e p$ and $\sigma \in C$ such that $\sigma(s)=r \in$ Rep. Consider a state $s$ where $M_{j, l}$ is the master elect. In this case $\sigma_{i, l}(s)$ is the state where $M_{j, 1}$ is the master elect. Hence all conditions for applying the multiple representative theory are satisfied. The table of experimental results is shown below.

\begin{tabular}{||l|c|c|c|c|}
\hline \hline $\begin{array}{l}\text { System } \\
\text { Config }\end{array}$ & Time & $\begin{array}{c}\text { BDD } \\
\text { size }\end{array}$ & $\begin{array}{c}\text { Time } \\
(\text { Symm })\end{array}$ & $\begin{array}{c}\text { BDD size } \\
\text { (Symm) }\end{array}$ \\
\hline $10 \mathrm{~m}$ & 163.41 & 369,705 & 37.04 & 27,671 \\
\hline $12 \mathrm{~m}$ & 487.56 & 921,034 & 43.12 & 36,135 \\
\hline $10 \mathrm{~m} 10 \mathrm{~m}$ & 1171.85 & 932,429 & 126.17 & 73,514 \\
\hline $12 \mathrm{~m} 12 \mathrm{~m}$ & - & - & 198.195 & 93,094 \\
\hline \hline
\end{tabular}

The property that was checked was that only one module is picked master elect. The first column shows the system configuration. For example, $10 \mathrm{~m}$ is the configuration with 10 modules having the same priority number. $10 \mathrm{~m} 10 \mathrm{~m}$ denotes the configuration where the first 10 modules have a higher priority number than the last 10 modules (there are 20 modules in all). Columns 2 and 3 shows the time (in seconds) and the maximum BDD size encountered during checking the temporal property without using symmetry. The experiments were run on an Sun ULTRA SPARC. The last column shows the same statistic with using symmetry. The symbol - means that we were unable to check the property in the time allowed. As can be seen from the table we get considerable savings by using symmetry.

\section{Conclusion}

In this paper we investigated various problems associated with exploiting symmetry in model checking. We also provided ways of deriving symmetry for shared variable concurrent programs. An important research problem will be to take some existing hardware description languages and derive symmetry information statically from the system descriptions written in that language. Ideas presented in section 3 are applicable in this context. This paper also makes connection between exploiting symmetry in model checking and computational group theory. An important research direction will be to use some of the powerful techniques available in the computational group theory literature in the model checking domain. It will be also very useful to apply some of the symmetry ideas on industrial size examples. 


\section{References}

1. L. Babai and E. Luks. Canonical labeling of graphs. In Proceedings of the 15th ACM STOC, 1983.

2. J. R. Burch, E. M. Clarke, K. L. McMillan, D. L. Dill, and L. J. Hwang. Symbolic model checking: $10^{20}$ states and beyond. 98(2):142-170, June 1992.

3. E. Clarke, R. Enders, T. Filkorn, and S. Jha. Exploiting symmetry in temporal logic model checking. Formal Methods in System Design, 9(1/2):77-104, 1996.

4. E. M. Clarke, E. A. Emerson, and A. P. Sistla. Automatic verification of finitestate concurrent systems using temporal logic specifications. ACM Trans. Prog. Lang. Syst., 8(2):244-263, Apr. 1986.

5. P. Curie. Sur la symétrie dans les phénomènons physiques, symétrie d'un champ électrique magnétique. J. Physics (3rd, ser.), 3:393-415, 1894.

6. E. Emerson and A. Sistla. Symmetry and model checking. Formal Methods in System Design, 9(1/2):105-130, 1996.

7. M. Furst, J. Hopcroft, and E. Luks. Polynomial-time algorithms for permutations groups. In Proceedings of the 21st Annual Symposium on Foundations of Computer Science, 1980.

8. C. Hoffman. Group Theoretic Algorithms and Graph Isomorphism, volume 697 of Lecture Notes in Computer Science. Springer-Verlag, Berlin, 1982.

9. T. Hungerford. Algebra. Springer-Verlag, 1980.

10. IEEE Computer Society. IEEE Standard for Futurebus+-Logical Protocol Specification, Mar. 1992. IEEE Standard 896.1-1991.

11. C. Ip and D. Dill. Better verification through symmetry. Formal Methods in System Design, 9(1/2):41-76, 1996.

12. K. Jensen. Condensed state spaces for symmetrical coloured petri nets. Formal Methods in System Design, 9(1/2):7-40, 1996.

13. E. Luks. Permutation groups and polynomial-time computation. In Workshop on Groups and Computation, volume 11 of Dimacs. American Mathematical Society, Oct. 1991.

14. K. L. McMillan. Symbolic Model Checking: An Approach to the State Explosion Problem. PhD thesis, Carnegie Mellon University, 1992. 